\title{
COMMENTARY
}

\section{An exploration of psychopathology for clinical practice ${ }^{\dagger}$}

\author{
Abdi Sanati (1)
}

Abdi Sanati is a consultant psychiatrist with East London NHS Foundation Trust, working in Newham Centre for Mental Health, a psychiatric intensive care unit in London. He is the chair of the Philosophy Special Interest Group at the Royal College of Psychiatrists, London, UK.

Correspondence Dr Abdi Sanati. Email: abstraxion@hotmail.com

First received 14 Apr 2020

Final revision 13 May 2020

Accepted 18 May 2020

\section{Copyright and usage}

The Authors 2020

${ }^{\dagger}$ Commentary on... The importance of embedding psychopathology and phenomenology in our clinical practice and training in psychiatry. See this issue.

\section{SUMMARY \\ Psychopathology is an integral part of the theory and practice of psychiatry. This commentary explores the importance of psychopathology, especially with regard to clinical practice. The concept of lifeworld is introduced as an important component of attributing meaning to a person's experience of mental disorder. It is also argued that the advance of neuroscience does not pre- clude the importance of psychopathology.}

\section{KEYWORDS}

Psychopathology; phenomenology; lifeworld; psychiatric interview; neuroscience.

Psychiatry is seen as a branch of medicine specialised in the disorders of the mind. It deals with morbid psychological experiences (Oyebode 2018). Professionals other than psychiatrists work in this field, but I believe that there are certain skills specific to psychiatry that cannot be replicated in other areas. One of these skills, I argue, is knowledge of psychopathology, which, in my opinion, is integral to the expertise of psychiatry as a profession. Phenomenological psychopathology is one of the main skills unique to psychiatry. Without it, psychiatry will be diluted and psychiatrists will be deprived of one of their most important tools to help patients. Chakraborty has provided an excellent overview of the discipline of psychopathology and the need for it (Chakraborty 2020, this issue). In what follows I aim to share my arguments for psychopathology.

Psychopathology has been defined as the 'systematic study of abnormal experience, cognition and behaviour - the products of a disordered mind' (Oyebode 2018: p. 4). Phenomenology may be seen as an enquiry into a person's conscious and intellectual processes without any preconceptions about their causes (Oyebode 2018: p. 4). Phenomenology has a long history in philosophy and has been associated with Edmund Husserl, Martin Heidegger and Maurice Merleau-Ponty, among others. The literature on phenomenology is rich. Phenomenology has been used in psychopathology by Karl Jaspers, although it is important to note that Jaspers has not been the only figure in psychopathology - a detailed history can be found in Berrios (1996).

\section{Psychopathology as a method}

In identifying different ways in which the term psychopathology is used, Stanghellini \& Aragona emphasise the importance of psychopathology as a method (Stanghellini 2016). It is much more than a collection of symptoms. In this specific methodology, mental symptoms and states of mind are described, delineated and differentiated from each other.

Psychopathology enables the clinician to understand what it is like to have a mental disorder. It also enables the clinician to get a grasp of the global experience of the patient. In other words, the clinician will understand the 'lifeworld' of the patient. Here is where the phenomenological aspects of psychopathology come forward. First, as stated above, an accurate description and subjective experience are elicited. In the next stage, which Stanghellini \& Aragona (2016: p. 4) call trance-phenomenal, features that underlie and constitute patients' experience, 'prior to and independent of the content', and the underlying structures or existential dimensions of the person's life are understood.

\section{The lifeworld}

One of the interesting concepts that is brought to psychopathology is the concept of the lifeworld. Stanghellini $\&$ Aragona define it as 'the province of reality inhabited by a given person' (Stanghellini 2016: p. 4). Zahavi (2019: p. 145) defines the lifeworld as the world we live in and take for granted in daily life, and the pre-theoretical world of experience that we do not question. It is important as science is mainly built on the pre-scientific evidence of this lifeworld (Zahavi 2019). The shared lifeworld will continue to influence science and also practices such as psychiatry. The patient's and doctor's shared lifeworld makes a basis on which a psychiatric interview takes place. The lifeworld provides a bedrock for criteria of validity and truth.

Examining the patient's lifeworld can be very helpful in illuminating the world of a person with mental illness. For example, Owen uses a lifeworld approach in investigating the phenomenon of bizarreness, which has an important role in 
assessing people with psychosis (Owen 2016). He argues that a lifeworld approach looks at psychopathology 'in terms of change to categories of human experience such as time, space, self, body, value, etc.' (Owen 2016: p. 227). It puts human experience at the centre of the investigation and contributes to truly patient-centred care. Extensive work has already been done to apply this approach to different mental illnesses (see Stanghellini 2019).

\section{The importance of phenomenological psychopathology}

The importance of phenomenological psychopathology manifests at different levels. At the level of diagnosis it is indispensable as, without truly understanding what the patient is experiencing, the psychiatrist cannot use any diagnostic criteria. Obtaining a detailed account of psychopathology requires spending time with the patient, knowing them and enquiring about their subjective experience. A patient is not a collection of symptoms that can be easily elicited using standard questionnaires. Understanding the subjective experience and its meaning for the patient is of utmost importance. To understand a person with depression psychiatrists need to go beyond asking simple questions about mood, energy and enjoyment. They need to know how it is for the person to feel like this. How it really feels for someone who says 'I am depressed', how this feeling changes the way they see themselves in the world and how the world appears to them. The numbing of colours and tastes might not be part of diagnostic criteria but understanding them through phenomenological enquiry helps psychiatrists to understand the person better. The use of psychopathology enables them to avoid the Procrustean error of stretching and trimming the patients' symptoms to fit the criteria (Stahghellini 2017). It is person oriented and, while it helps with diagnosis, the diagnostic process is not its main goal (Stanghellini 2016).

Just as psychiatry cannot be reduced to simply making a diagnosis on the basis of the ICD or DSM, phenomenological psychopathology, while being the cornerstone of making a diagnosis, is more than a diagnostic tool. It enables psychiatrists to make sense of the person's experience. While they use research to identify causal explanations, phenomenological psychopathology helps them to elicit reasons for the person's experience. This distinction has been explained in detail by Jaspers (1913). Jaspers distinguished causal explanations from an understanding of the patient that involves hermeneutics - understanding how mental states meaningfully emerge from each other. One clear indication of its use is in identifying delusions. Phenomenological psychopathology offers a more enriched understanding of delusional phenomena, which the use of rationality criteria does not.

Psychopathology also helps the psychiatrist to integrate diagnostic and therapeutic processes. The psychiatrist's aim is more than diagnosing the person; it extends to understanding the meaningfulness of the patient's existence and turning the interview into a therapeutic process. In my clinical experience, understanding the patient's experience has been an important step in building the therapeutic relationship which, in my opinion, is extremely important, even necessary, in any therapeutic process in psychiatry.

\section{Psychopathology and neuroscience}

In addition to averting psychiatry's reduction to diagnostic criteria, phenomenological psychopathology prevents psychiatry's reduction to neuroimaging. At present, there are no clear neuroimaging techniques to enable bedside diagnosis of mental illness. It has been argued that psychiatry could never be reduced to neuroscience - even if an accurate diagnostic neuroimaging test were developed, it would not preclude the importance of psychopathology, for the following reasons. First, as in other areas of medicine, diagnostic tests in psychiatry aid the diagnosis and are guided by thorough history-taking. Psychopathology will still be integral in guiding the investigations that have to be carried out. Second, there is no diagnostic test in psychiatry with $100 \%$ sensitivity and specificity. There will always be false positives and false negatives and it is the clinician's job to navigate through the results and, again, a thorough history and mental state examination using clear knowledge of psychopathology are essential tools in this. Third, to find the diagnostic tests psychiatrists do need a clear understanding of the symptoms that patients experience and that, in turn, would need clear knowledge of psychopathology.

\section{Final words}

Psychopathology is indeed one of the most interesting parts of psychiatry. It enriches the interaction with patients and, by focusing on their subjective experience, contributes to true patient-centred care. Unfortunately, in the current era of inadequate resources and excessive focus on targets, which has led to a form of assembly-line psychiatry, it is not always possible to practise it in the way it is supposed to be practised. Nevertheless, psychiatrists must not forget that it is one of their main unique skills and they ignore it at their peril.

\section{Declaration of interest}

None. 
An ICMJE form is in the supplementary material, available online at https://doi.org/10.1192/bja. 2020.37

\section{References}

Berrios G (1996) The History of Mental Symptoms: Descriptive Psychopathology Since the Nineteenth Century. Cambridge University Press.

Chakraborty N (2020) The importance of embedding psychopathology and phenomenology in clinical practice and training in psychiatry. BJPsych Advances, 26: this issue.

Jaspers K (1913) Allgemeine Psychopathologie. Reprinted (1963) as General Psychopathology: Vol. I (trans J Hoenig, MW Hamilton). Johns Hopkins University Press.
Owen GS (2016) The phenomenological approach to patients with bizarreness. In An Experiential Approach to Psychopathology: What is it Like to Suffer from Mental Disorders? (eds G Stanghellini, M Aragona): 221-30. Springer.

Oyebode F (2018) Sims' Symptoms in The Mind: Textbook of Descriptive Psychopathology: Textbook of Descriptive Psychopathology (6th edn). Elsevier.

Stahghellini G, Mancini M (2017) The Therapeutic Interview in Mental Health: A Values-Based and Person-Centred Approach. Cambridge University Press.

Stanghellini G, Aragona M (eds) (2016) An Experiential Approach to Psychopathology: What is Like to Suffer from Mental Disorders? Springer.

Stanghellini G, Broome M, Fernandez A, et al (eds) (2019) The Oxford Handbook of Phenomenological Psychopathology. Oxford University Press.

Zahavi D (2019) Phenomenology: The Basics. Routledge. 